\title{
Ventricular Fibrillation in a Left Ventricular Assist Device Patient: Can the echocardiogram be misleading?
}

\author{
Manavotam Singh ${ }^{1}$, Nitin Malik ${ }^{1}$, Vijaywant Brar ${ }^{1}$, Cyrus Hadadi ${ }^{1}$, and Phillip Lam ${ }^{1}$ \\ ${ }^{1}$ MedStar Washington Hospital Center
}

October 15, 2020

\begin{abstract}
Sustained ventricular tachycardia and ventricular fibrillation (VF) are life-threatening arrhythmias which remain highly prevalent in patients with advanced heart failure. These ventricular arrhythmias may impair the support provided by CF-LVADs and lead to frequent hospitalizations, antiarrhythmic medication use, external defibrillations, and need for heart transplantation. We report a case in which a patient with a CF-LVAD and an ICD at end-of-life (EOL) presented with asymptomatic low-flow alarms and was found to have VF of unknown duration. Unique in our case was the presence of organized contractility and rhythmic opening of the mitral valve on echocardiogram despite VF on ECG.
\end{abstract}

\section{Background}

Continuous-Flow Left Ventricular Assist Devices (CF-LVAD) provide circulatory support to patients with advanced heart failure. These devices are traditionally used as a temporary bridge to recovery, bridge to cardiac transplantation, or as destination therapy (DT). Compared to medical management alone, LVADs improve survival ${ }^{1-3}$ and quality of life ${ }^{4,5}$ for patients with severe heart failure. The incidence of ventricular arrhythmias in patients with CF-LVADs are relatively high and range anywhere from 22-59\%. ${ }^{6}$ Sustained ventricular tachycardia and ventricular fibrillation are life-threatening arrhythmias which remain highly prevalent in patients with advanced heart failure. ${ }^{7}$ In particular, ventricular arrhythmias may impair hemodynamic support provided by CF-LVADs and lead to greater frequency in hospitalizations, antiarrhythmic therapies, external defibrillations, and urgent need for heart transplantation. ${ }^{13,14}$

We report a case in which a patient with a CF-LVAD and an implantable cardioverter defibrillator (ICD) at end of life (EOL) presented with asymptomatic low-flow alarms and was found to have ventricular fibrillation of unknown duration yet demonstrated organized contractility on echocardiography.

\section{Case}

A 58-year-old male with medical history of non-ischemic cardiomyopathy and American College of Cardiology / American Heart Association Stage D heart failure status post implantation of a HeartWare LVAD (HWLVAD; Medtronic, Minneapolis, MN) as well as an ICD presented to the emergency room with multiple low-flow alarms over two days duration. On initial evaluation, the patient was awake and conversant with a mean arterial blood pressure (MAP) of $90 \mathrm{~mm}$ of $\mathrm{Hg}$, oxygen saturation of $97 \%$ on room air, and heart rate that could not be automatically determined on telemetry. His lungs were clear to auscultation and he had a normal LVAD hum over the precordium. His HW-LVAD interrogation displayed a flow rate of 2.9 liters/minute with multiple low-flow alarms (baseline flow rate of approximately 4 liters/minute) using a power of 3.8 watts at a speed of 2600 revolutions per minute (RPM). Flow waveform qualitatively appeared significantly dampened. Initial electrocardiogram (ECG) demonstrated disorganized rhythm (ECG 1) which was thought to be due to artifact from the LVAD, as it was markedly different from his prior ECGs. In order to confirm the underlying arrythmias, his ICD was interrogated, which was found to be at EOL 
and was unable to record any events. A bedside echocardiogram was performed, which showed rhythmic hypokinetic contraction of the left ventricle and regular opening and closing of the mitral valve (image 1 , image 2). The apical 4-chamber view with perflutren lipid contrast (clip 1) and a parasternal long image without contrast (clip 2) also showed organized ventricular contractility. Therefore, emergent defibrillation was deferred as it was unclear whether the arrhythmia was atrial fibrillation (AF) distorted by artifact or ventricular fibrillation $(\mathrm{VF})$ and because patient remained hemodynamically stable. Initial laboratory work was significant for serum creatinine of $2.7 \mathrm{mg} / \mathrm{dL}$ from baseline of 1.5-1.7 mcg/dL, potassium level of $4.9 \mathrm{mmol} / \mathrm{L}$ and magnesium level of $1.7 \mathrm{mg} / \mathrm{dL}$. The patient received intravenous infusion of normal saline, cardiac electrophysiology was consulted, and the patient was admitted to the Intermediate Care Unit (IMC).

Subsequently low-flow alarms recurred without symptoms and MAP at that time remained preserved. Decreased flow rates were intermittently associated with loss of pulsatility on the LVAD monitor and drops in LVAD speed, concerning for suction events. His HW-LVAD speed was decreased to 2160 RPMs; however, due to persistence of low flow alarms and loss of pulsatility on the LVAD monitor, the patient was defibrillated at $200 \mathrm{~J}$ with subsequent conversion of irregular disorganized activity to normal sinus rhythm (ECG 2). Post defibrillation, his HW-LVAD flow rate improved to 3.5 liters/min along with restoration of baseline pulsatility. LVAD speed was increased back to 2600 RPMs, an intravenous amiodarone infusion was started, and the patient was transferred to the intensive care unit for closer monitoring. Subsequently, the patient underwent a ventricular tachycardia induction study which confirmed VF with successful defibrillation testing. His ICD generator was replaced.

\section{Discussion}

Ventricular arrhythmias (VAs) are frequently seen after LVAD placement, with ventricular fibrillation being the most common. ${ }^{6}$ Ventricular fibrillation is a terminal cardiac arrhythmia characterized by disorganized, high-frequency ventricular contractions that result in diminished cardiac output and hemodynamic collapse. Risk factors of VF include cardiomyopathies, electrolyte abnormalities, acidosis, hypoxemia, and ischemia. ${ }^{8,9,10}$ Pre-LVAD ventricular arrhythmias are also a known risk factor for ventricular arrhythmia post-LVAD implantation, reflective of the fact that LVADs do not necessarily reverse underlying arrhythmogenicity. ${ }^{13}$

Patients are typically equipped with an ICD in order to terminate sustained VAs, but our patient's ICD was at EOL and therefore was unable to terminate VF. Patients with CF-LVAD may tolerate otherwise life-threatening arrhythmias for certain period of time with no or minimal symptoms as the device supports their native cardiac function. ${ }^{11,12}$ However, as seen in our case, it is very important to defibrillate even asymptomatic patients with ongoing VAs to sinus (or paced) rhythm as soon as possible, since their ability to maintain adequate hemodynamic stability remains tenuous and can lead to right ventricular failure due to prolonged dysrhythmia. ${ }^{17}$

Noteworthy in our case was the presence of organized contractility and rhythmic opening of the mitral valve on echocardiogram despite ventricular fibrillation on ECG. While some cardiac motion and valve opening could be explained by the negative pressure generated by the device, ventricular fibrillation is generally thought to manifest as asynchronous ventricular activity. The fact that organized activity appeared on echocardiogram suggests that the echocardiographic findings may appear incongruous with ECG findings of ventricular fibrillation. Gray et al. described spatio-temporal patterns of electrical activity of isolated blood-perfused dog, rabbit, and sheep hearts during ventricular fibrillation and found that there was spatial and temporal patterning rather than total chaotic electrical activity. ${ }^{15}$ Furthermore, clinical and simulation data of human hearts suggest that VF in humans is driven by fewer reentrant sources and organized by fewer rotors compared to VF in animal models. ${ }^{16}$ It is possible that these features of VF in human hearts contributed to the seemingly discordant findings of VF on ECG yet organized contraction on non-invasive imaging.

\section{Conclusion}

CF-LVAD patients may tolerate life-threatening VAs for certain period of time with no or minimal symptoms 
as the device supports their native cardiac function. These patients with CF-LVADs should be carefully evaluated for arrhythmias when presenting with any type of illness. If VF is suspected on ECG in a CFLVAD patient, presence of organized cardiac activity on echocardiogram can be misleading in context of the patient's full clinical picture. It is important to take CF-LVAD parameters, including low flow alarms and changes in the flow waveform, into consideration as data points in the decision-making process as management with early cardiac defibrillation may be warranted.

\section{References}

1. Slaughter MS, Rogers JG, Milano CA, et al. Advanced heart failure treated with continuous-flow left ventricular assist device. N Engl J Med 2009;361:2241e2251.

2. Miller LW, Pagani FD, Russell SD, et al.: Use of a continuous-flow device in patients awaiting heart trans-plantation. N Engl J Med 2007;357:885-896.

3. Aaronson KD, Slaughter MS, Miller LW, et al.: Use of an intrapericardial, continuous-flow, centrifugal pump in patients awaiting heart transplantation. Circulation 2012;125:3191-3200.

4. McIlvennan CK, Magid KH, Ambardekar AV, et al.: Clinical outcomes after continuous-flow left ventricular assist device: A systematic review. Circ Heart Fail 2014;7:1003-1013.

5. Rogers JG, Aaronson KD, Boyle AJ, et al.: Continuous flow left ventricular assist device improves functional ca-pacity and quality of life of advanced heart failure patients. J Am Coll Cardiol 2010;55:18261834 .

6. Ahmed A, Amin M, Boilson BA et al: Ventricular arrhythmias in patients with left ventricular assist device (LVAD). Curr Treat Options Cardio Med, 2019; 21(11): 75

7. Saxon LA, BristowMR, Boehmer J, et al. Predictors of sudden cardiac death and appropriate shock in the Comparison of Medical Therapy, Pacing, and Defibrillation in Heart Failure (COMPANION) trial.Circulation 2006;114:2766-72.

8. Prinzing A, Herold U, Berkefeld A, Krane M, Lange R, Voss B. Left ventricular assist devices-current state and perspectives. J Thorac Dis. 2016 Aug;8(8):E660-6. doi: 10.21037/jtd.2016.07.13. PMID: 27621895; PMCID: PMC4999658.

9. Kilic A, Acker MA, Atluri P. Dealing with surgical left ventricular assist device complications. J Thorac Dis. 2015 Dec;7(12):2158-64. doi: 10.3978/j.issn.2072-1439.2015.10.64. PMID: 26793336; PMCID: PMC4703654.

10. Bujo C, Amiya E, Hatano M, Tsuji M, Maki H, Hosoya Y, Fujii E, Kamon T, Kojima T, Nawata K, Kinoshita O, Kimura M, Ono M, Komuro I. Clinical impact of newly developed atrial fibrillation complicated with longstanding ventricular fibrillation during left ventricular assist device support: A case report. BMC Cardiovasc Disord. 2019 Jun 21;19(1):151. doi: 10.1186/s12872-019-1132-1. PMID: 31226943; PMCID: PMC6588891.

11. Patel P, Williams JG, Brice JH. Sustained ventricular fibrillation in an alert patient: preserved hemodynamics with a left ventricular assist device. Prehospital Emerg Care Off J Natl Assoc EMS Physicians Natl Assoc State EMS Dir 011;15(4):533-6.

12. Busch MC, Haap M, Kristen A, Haas CS. Asymptomatic sustained ventricular fibrillation in a patient with left ventricular assist device. Ann Emerg Med 2011;57(1):25-8

13. Yap et al. "Ventricular Arrhythmias in Patients With a Continuous-Flow Left Ventricular Assist Device" JACC (Journal of the American College of Cardiology), 2016-07-19, Volume 68, Issue 3, Pages 323-325, Copyright (C) 2016 American College of Cardiology Foundation

14. Raasch et al "Epidemiology, management, and outcomes of sustained ventricular arrhythmias after continuous-flow left ventricular assist device implantation" American Heart Journal, 2012-09-01, Volume 164, Issue 3, Pages 373-378, Copyright (C) 2012 Mosby, Inc.

15. Gray RA, Pertsov AM, Jalife J. Spatial and temporal organization during cardiac fibrillation. Nature. 1998 Mar 5;392(6671):75-8. doi: 10.1038/32164. Erratum in: Nature 1998 May 14;393(6681):191. PMID: 9510249.

16. Kirsten et al. Organization of Ventricular Fibrillation in the Human Heart, https://doi.org/10.1161/CIRCRESAHA.107.150730Circulation Research. 2007;100:e87-e101 
17. Baldwin ACW, Gemmato CJ, Sandoval E, Cohn WE, Morgan JA, Frazier OH. Tolerance of Sustained Ventricular Fibrillation During Continuous-Flow Left Ventricular Assist Device Support. Tex Heart Inst $J$. 2017;44(5):357-360. Published 2017 Oct 1. doi:10.14503/THIJ-16-5879

Figure Legend:

1. ECG 1: Initial ECG obtained on presentation to the hospital demonstrating disorganized activity. ECG 2: Post defibrillation ECG demonstrating normal sinus rhythm.

2. Image 1: Pulsed-Wave doppler of mitral inflow shows rhythmic ventricular filling. Image 2: M-mode of the mitral valve leaflets reveals rhythmic opening and closing of valve leaflets.

3. Clip 1: An apical 4-chamber view with perflutren lipid microsphere contrast shows organized contractility despite ventricular fibrillation.

4. Clip 2: A parasternal long image without contrast similarly reveals organized contractility.

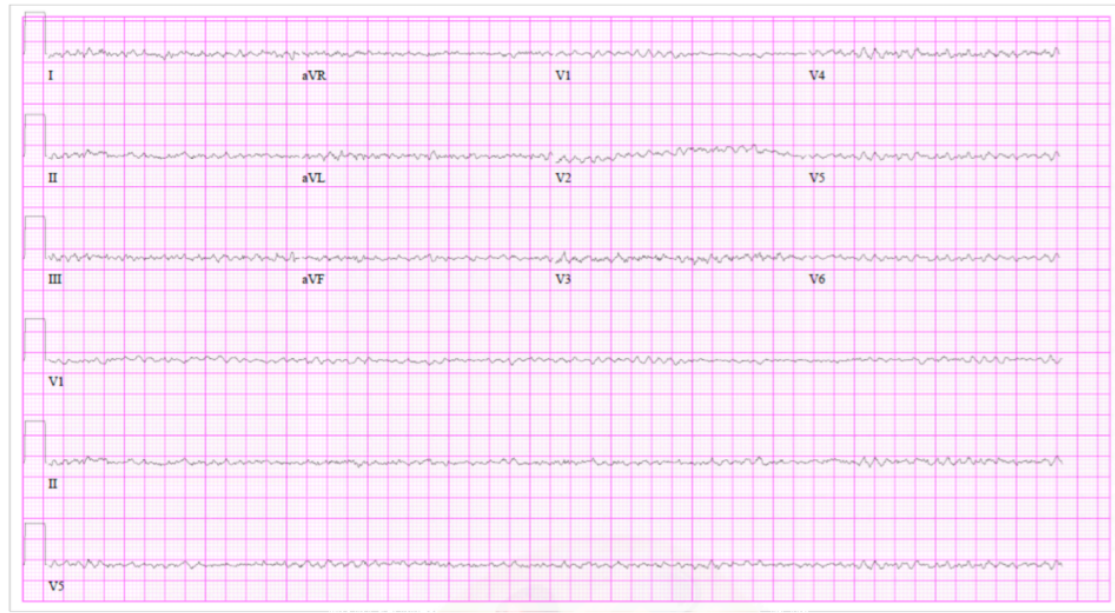

ECG 1: Initial ECG obtained on presentation to the hospital demonstrating disorganized activity.

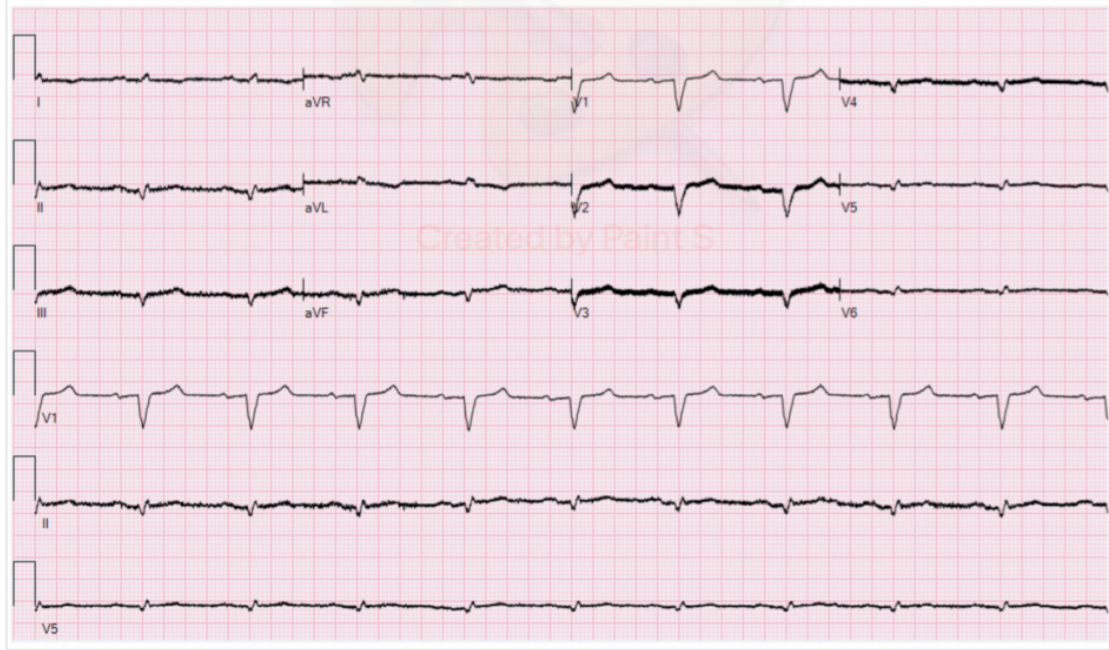

ECG 2: Post defibrillation ECG demonstrating normal sinus rhythm. 


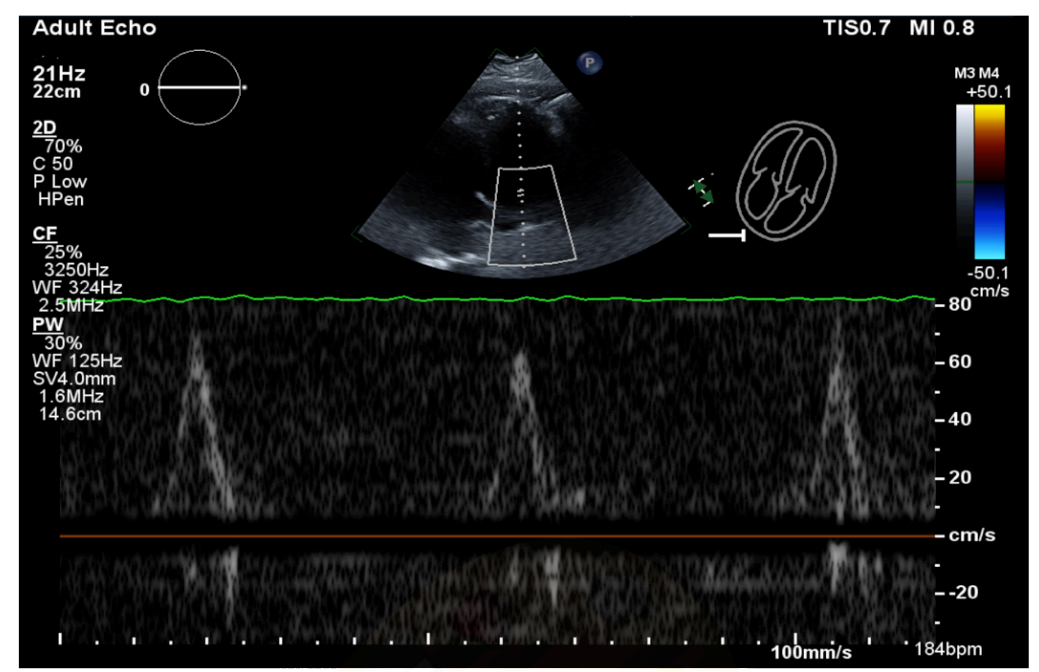

Image 1: Pulsed-Wave Doppler of mitral inflow shows rhythmic ventricular filling.

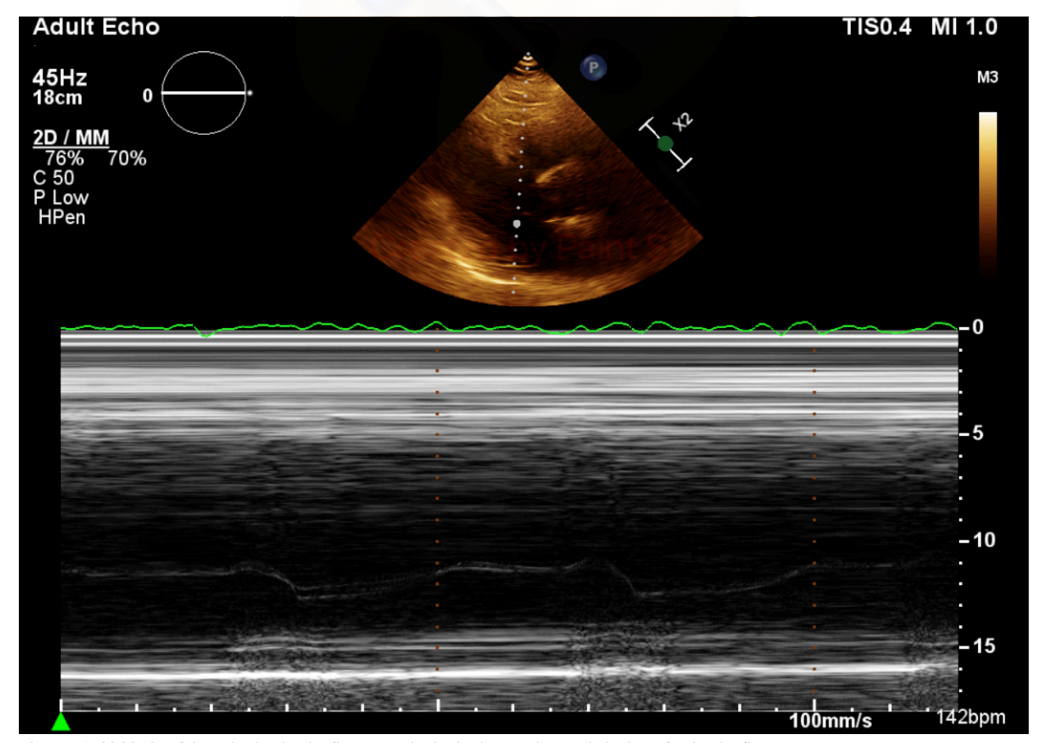

Image 2: M-Mode of the mitral valve leaflets reveals rhythmic opening and closing of valve leaflets.

\section{Hosted file}

Clip 1-A4cContrast.avi available at https://authorea.com/users/367322/articles/486798ventricular-fibrillation-in-a-left-ventricular-assist-device-patient-can-theechocardiogram-be-misleading

\section{Hosted file}

Clip 2-ParasternalLongWOcontrast.avi available at https://authorea.com/users/367322/articles/ 486798-ventricular-fibrillation-in-a-left-ventricular-assist-device-patient-can-theechocardiogram-be-misleading 